\title{
New Fermions at the LHC and Mass of the Higgs Boson
}

\author{
Ilia Gogoladze1느, Bin He 2 and Qaisar Shafi \\ Bartol Research Institute, Department of Physics and Astronomy, \\ University of Delaware, Newark, DE 19716, USA
}

\begin{abstract}
Unification at $M_{\mathrm{GUT}} \sim 3 \times 10^{16} \mathrm{GeV}$ of the three Standard Model (SM) gauge couplings can be achieved by postulating the existence of a pair of vectorlike fermions carrying SM charges and masses of order $300 \mathrm{GeV}-1 \mathrm{TeV}$. The presence of these fermions significantly modifies the vacuum stability and perturbativity bounds on the mass of the SM Higgs boson. The new vacuum stability bound in this extended SM is estimated to be $117 \mathrm{GeV}$, to be compared with the SM prediction of about $128 \mathrm{GeV}$. An upper bound of $190 \mathrm{GeV}$ is obtained based on perturbativity arguments. The impact on these predictions of type I seesaw physics is also discussed. The discovery of a relatively 'light' Higgs boson with mass $\sim 117 \mathrm{GeV}$ could signal the presence of new vectorlike fermions within reach of the LHC.
\end{abstract}

\footnotetext{
${ }^{1}$ E-mail: ilia@physics.udel.edu

On leave of absence from: Andronikashvili Institute of Physics, GAS, 380077 Tbilisi, Georgia.

${ }^{2}$ E-mail: hebin@udel.edu
} 


\section{Introduction}

The discovery of the Standard Model (SM) Higgs boson is arguably the single most important mission for the LHC. Under a somewhat radical assumption that the next energy frontier lies at the reduced Planck scale $\left(M_{P} \simeq 2.4 \times 10^{18} \mathrm{GeV}\right)$, it has been found that the SM Higgs boson mass lies in the range $128 \mathrm{GeV} \lesssim m_{H} \lesssim 175 \mathrm{GeV}$ [1]. Here the lower bound of $128 \mathrm{GeV}$ on $m_{H}$ derives from arguments based on the stability of the SM vacuum. More precisely, that the Higgs quartic coupling does not become negative at any scale between $M_{Z}$ and $M_{P}$. The upper bound of $175 \mathrm{GeV}$ or so on $m_{H}$ stems from the requirement that the Higgs quartic coupling remains perturbative and does not exceed $4 \pi$, say, during its evolution between $M_{Z}$ and $M_{P}$. Thus, it would appear that discovery of a relatively 'light' Higgs boson (with mass well below $128 \mathrm{GeV}$ ) may signal the presence of physics beyond the SM.

Supersymmetry is by far the most compelling extension of the SM and its minimal realization (MSSM) predicts a relatively 'light' SM-like Higgs boson with mass $\lesssim 130 \mathrm{GeV}$. However, in the light of LHC, plausible alternatives to supersymmetry deserve careful investigation. For instance, it was shown in [2] that the new physics between $M_{Z}$ and $M_{P}$ associated with type II seesaw [3] around TeV scale or higher can yield a 'light' Higgs boson with mass $\gtrsim 114.4 \mathrm{GeV}$, the LEP II bound. A 'light' Higgs boson is also realized in scenarios of gauge-Higgs unification with a compactification scale below $M_{P}$ [4].

In this paper we revisit another extension of the SM, proposed several years ago, in which new $\mathrm{TeV}$ scale vectorlike fermions are introduced in order to implement unification at some scale $M_{\mathrm{GUT}}$ of the three SM gauge couplings [5]. The new vectorlike fermions carry SM gauge quantum numbers and their presence therefore modifies the SM Higgs mass bounds based on vacuum stability and perturbativity arguments. In particular, by including only a pair of vectorlike fermions for which case $M_{\mathrm{GUT}} \simeq 3 \times 10^{16} \mathrm{GeV}$, the vacuum stability bound can be lowered from its conventional value of around $128 \mathrm{GeV}$ to a significantly lower value of about $117 \mathrm{GeV}$. To keep the discussion as realistic as possible, we also study the possible impact neutrino oscillation physics could have on the Higgs mass predictions. We employ type I seesaw for these considerations [6]. Note that a more complicated scenario containing several new particles (including scalars) can yield $M_{\mathrm{GUT}} \simeq M_{P}$, with a vacuum stability bound as low as $114 \mathrm{GeV}$.

This letter is organized as follows. In Section 2, we investigate the impact of new vectorlike fermions on the vacuum stability and perturbativity Higgs mass bounds. In Section 3, we discuss how neutrino physics utilizing type I seesaw can affect the vacuum stability and perturbativity bounds. Our conclusion is outlined in section 4 . 


\section{New Fermions and the Higgs Boson Mass}

We introduce the following vectorlike fermions to achieve gauge coupling unification:

$$
Q\left(3,2, \frac{1}{6}\right)+\bar{Q}\left(\overline{3}, 2,-\frac{1}{6}\right)+D\left(3,1, \frac{1}{3}\right)+\bar{D}\left(\overline{3}, 1,-\frac{1}{3}\right)
$$

where the brackets contain the $S U(3)_{c} \times S U(2)_{L} \times U(1)_{Y}$ quantum numbers of the new particles. The SM Lagrangian is supplemented by additional terms, and the relevant ones are given by

$$
\mathcal{L}_{\text {new }}=-\kappa_{1} \bar{Q} \bar{D} \Phi^{c}-\kappa_{2} Q D \Phi-y_{1}^{i} Q d_{i}^{c} \Phi-y_{2}^{i} q_{i} D \Phi-y_{3}^{i} Q u_{i}^{c} \Phi^{c}-M_{F}(\bar{Q} Q+\bar{D} D)+\text { h.c. }
$$

where $\Phi$ denotes the SM higgs doublet, $\Phi^{c} \equiv i \sigma_{2} \Phi^{*}$ its charge conjugate, and we employ the standard notation $q_{i}, u_{i}^{c}, d_{i}^{c}$ for the SM quarks, with $i=1,2,3$. The parameters $y_{1,2,3}^{i}$ and $\kappa_{1,2}$ are dimensionless couplings. We assume, for simplicity, that the new fermions have a common vectorlike mass $M_{F}$. As pointed out in [7], most of the $y_{1,2,3}^{i}$ couplings have to be very small due to constraints from the precision electroweak data. To accommodate this, we will assume that the couplings $y_{1,2,3}^{i}$ are sufficiently small so that they do not give a significant contribution in the RGE analysis. However, the $y^{i}$ 's allow the new fermions to decay into the SM particles, without creating any cosmological problems.

There are constraints on the $\kappa_{1,2}$ couplings and the masses of the new matter fields. The most important ones arise from the $S$ and $T$ parameters which severely limit the number of additional chiral generations. Consistent with these constraints, one should therefore add new matter which is predominantly vectorlike. In the limit where the vectorlike mass $M_{F}$ is much heavier than the chiral mass term (arising from Yukawa coupling to the Higgs doublets), the contribution to the $T$ parameter from a single chiral fermion is given by [8]

$$
\delta T \approx \frac{N\left(\kappa_{i} v\right)^{2}}{10 \pi \sin ^{2} \theta_{W} m_{W}^{2}}\left[\left(\frac{\kappa_{i} v}{M_{V}}\right)^{2}+O\left(\frac{\kappa_{i} v}{M_{V}}\right)^{4}\right]
$$

where $\kappa_{i}, i=1,2$, are the Yukawa couplings in Eq. (2), $v=246.2 \mathrm{GeV}$ is the vacuum expectation value (VEV) of the Higgs field, and $N$ counts the number of additional $\mathrm{SU}(2)$ doublet pairs, which in our case is 3 . From the precision electroweak data $T \leq 0.06(0.14)$ at 95\% CL for $m_{H}=117 \mathrm{GeV}(300 \mathrm{GeV})$ [9]. We will take $\delta T<0.1$ as a conservative bound for our analysis. We see from Eq. (3) that with $M_{F} \sim 500 \mathrm{GeV}$, the Yukawa couplings $\kappa_{i}$ can be $O(1)$.

For the SM gauge coupling we employ the two renormalization group equation (RGE) [10] :

$$
\frac{d g_{i}}{d \ln \mu}=\frac{b_{i}}{16 \pi^{2}} g_{i}^{3}+\frac{g_{i}^{3}}{\left(16 \pi^{2}\right)^{2}}\left(\sum_{j=1}^{3} B_{i j} g_{j}^{2}-C_{i}^{t} y_{t}^{2}\right)
$$


where $g_{i}(i=1,2,3)$ are the SM gauge couplings and $y_{t}$ is the top Yukawa coupling,

$$
b_{i}^{S M}=\left(\frac{41}{10},-\frac{19}{6},-7\right), \quad B_{i j}^{S M}=\left(\begin{array}{ccc}
\frac{199}{50} & \frac{27}{10} & \frac{44}{5} \\
\frac{9}{10} & \frac{35}{6} & 12 \\
\frac{11}{10} & \frac{9}{2} & -26
\end{array}\right), \quad C_{i}^{t}=\left(\frac{17}{10}, \frac{3}{2}, 2\right) .
$$

For a renormalization scale $\mu>M_{F}$, the beta function for gauge couplings receives an additional contribution from the vectorlike fermions,

$$
b_{i}^{\prime}=\left(\frac{2}{5}, 2,2\right), \quad B_{i j}^{\prime}=\left(\begin{array}{ccc}
\frac{3}{50} & \frac{3}{10} & \frac{8}{5} \\
\frac{1}{10} & \frac{49}{2} & 8 \\
\frac{1}{5} & 3 & \frac{114}{3}
\end{array}\right), \quad C_{i}^{\kappa_{1}}=C_{i}^{\kappa_{2}}=\left(\frac{1}{2}, \frac{3}{2}, 2\right),
$$

where $C_{i}^{\kappa_{1}}$ and $C_{i}^{\kappa_{2}}$ stand for the contribution which is proportional to the $\kappa_{i}$ coupling in the two loop RGE for gauge couplings.

For the top Yukawa coupling, we have [10]

$$
\frac{d y_{t}}{d \ln \mu}=y_{t}\left(\frac{1}{16 \pi^{2}} \beta_{t}^{(1)}+\frac{1}{\left(16 \pi^{2}\right)^{2}} \beta_{t}^{(2)}\right) \text {. }
$$

Here the one-loop contribution is

$$
\beta_{t}^{(1)}=\frac{9}{2} y_{t}^{2}-\left(\frac{17}{20} g_{1}^{2}+\frac{9}{4} g_{2}^{2}+8 g_{3}^{2}\right),
$$

while the two-loop contribution is given by

$$
\begin{aligned}
\beta_{t}^{(2)}= & -12 y_{t}^{4}+\left(\frac{393}{80} g_{1}^{2}+\frac{225}{16} g_{2}^{2}+36 g_{3}^{2}\right) y_{t}^{2}+\frac{1187}{600} g_{1}^{4}-\frac{9}{20} g_{1}^{2} g_{2}^{2} \\
& +\frac{19}{15} g_{1}^{2} g_{3}^{2}-\frac{23}{4} g_{2}^{4}+9 g_{2}^{2} g_{3}^{2}-108 g_{3}^{4}+\frac{3}{2} \lambda^{2}-6 \lambda y_{t}^{2} .
\end{aligned}
$$

In solving Eq. (17), the initial top Yukawa coupling at $\mu=M_{t}$ is determined from the relation between the pole mass and the running Yukawa coupling [11, 12],

$$
M_{t} \simeq m_{t}\left(M_{t}\right)\left(1+\frac{4}{3} \frac{\alpha_{3}\left(M_{t}\right)}{\pi}+11\left(\frac{\alpha_{3}\left(M_{t}\right)}{\pi}\right)^{2}-\left(\frac{m_{t}\left(M_{t}\right)}{2 \pi v}\right)^{2}\right)
$$

with $y_{t}\left(M_{t}\right)=\sqrt{2} m_{t}\left(M_{t}\right) / v$ and $\alpha_{3} \equiv g_{3}^{2} / 4 \pi$. Here, the second and third terms in parentheses correspond to one- and two-loop QCD corrections, respectively, while the fourth term comes from the electroweak corrections at one-loop level. The numerical values of the third and fourth terms are comparable (their signs are opposite). The electroweak corrections at two-loop level and the three-loop QCD corrections are both comparable and of sufficiently small magnitude [12] to be safely ignored. 
For a renormalization scale $\mu>M_{F}$, according to the Eq. (2), the beta function for the top Yukawa coupling receives an additional contribution at one loop level as follows:

$$
\delta \beta_{t}^{(1)}=3\left(\kappa_{1}^{2}+\kappa_{2}^{2}\right)
$$

and the additional two loop contributions are

$$
\delta \beta_{t}^{(2)}=\left(\frac{5}{8} g_{1}^{2}+\frac{45}{8} g_{2}^{2}+20 g_{3}^{2}\right)\left(\kappa_{1}^{2}+\kappa_{2}^{2}\right)-\frac{27}{4}\left(\kappa_{1}^{4}+\kappa_{2}^{4}\right)-\frac{27}{4} y_{t}^{2}\left(\kappa_{1}^{2}+\kappa_{2}^{2}\right) .
$$

The one and two loop RGEs for the Yukawa couplings $\kappa_{1}$ and $\kappa_{2}$ are given by

$$
\frac{d \kappa_{1}}{d \ln \mu}=\kappa_{1}\left(\frac{1}{16 \pi^{2}} \beta_{\kappa_{1}}^{(1)}+\frac{1}{\left(16 \pi^{2}\right)^{2}} \beta_{\kappa_{1}}^{(2)}\right) \text {. }
$$

Here the one loop contribution is

$$
\beta_{\kappa_{1}}^{(1)}=-\frac{1}{4} g_{1}^{2}-\frac{9}{4} g_{2}^{2}-8 g^{3}+\frac{9}{2} \kappa_{1}^{2}+3 \kappa_{2}^{2}+3 y_{t}^{2},
$$

while the two-loop contribution is given by

$$
\begin{aligned}
\beta_{\kappa_{1}}^{(2)}= & -\frac{127}{600} g_{1}^{4}-\frac{23}{4} g_{2}^{4}-108 g_{3}^{4}-\frac{27}{20} g_{1}^{2} g_{2}^{2}+\frac{31}{15} g_{1}^{2} g_{3}^{2}+9 g_{2}^{2} g_{3}^{2}-6 \lambda \kappa_{1}^{2} \\
& +\left(\frac{85}{40} g_{1}^{2}+\frac{45}{8} g_{2}^{2}+20 g_{3}^{2}\right) y_{t}^{2}+\left(\frac{237}{80} g_{1}^{2}+\frac{225}{16} g_{2}^{2}+36 g_{3}^{2}\right) \kappa_{1}^{2}+\frac{3}{2} \lambda^{2} \\
& +\left(\frac{5}{8} g_{1}^{2}+\frac{45}{8} g_{2}^{2}+20 g_{3}^{2}\right) \kappa_{2}^{2}-12 \kappa_{1}^{4}-\frac{27}{4}\left(y_{t}^{4}+\kappa_{2}^{4}+y_{t}^{2} \kappa_{1}^{2}+\kappa_{1}^{2} \kappa_{2}^{2}\right) .
\end{aligned}
$$

The RGE for the Yukawa coupling $\kappa_{2}$ is obtained by making the replacement $\kappa_{1} \leftrightarrow \kappa_{2}$ in Eqs. (13)-(15). This follows from the various quantum numbers listed in Eq. (1). As previously mentioned, we are neglecting mixing terms involving the new vectorlike particles and the SM ones.

The RGE for the Higgs boson quartic coupling is given by [10]

$$
\frac{d \lambda}{d \ln \mu}=\frac{1}{16 \pi^{2}} \beta_{\lambda}^{(1)}+\frac{1}{\left(16 \pi^{2}\right)^{2}} \beta_{\lambda}^{(2)}
$$

with

$$
\beta_{\lambda}^{(1)}=12 \lambda^{2}-\left(\frac{9}{5} g_{1}^{2}+9 g_{2}^{2}\right) \lambda+\frac{9}{4}\left(\frac{3}{25} g_{1}^{4}+\frac{2}{5} g_{1}^{2} g_{2}^{2}+g_{2}^{4}\right)+12 y_{t}^{2} \lambda-12 y_{t}^{4}
$$

and

$$
\begin{aligned}
\beta_{\lambda}^{(2)}= & -78 \lambda^{3}+18\left(\frac{3}{5} g_{1}^{2}+3 g_{2}^{2}\right) \lambda^{2}-\left(\frac{73}{8} g_{2}^{4}-\frac{117}{20} g_{1}^{2} g_{2}^{2}-\frac{1887}{200} g_{1}^{4}\right) \lambda-3 \lambda y_{t}^{4} \\
& +\frac{305}{8} g_{2}^{6}-\frac{867}{120} g_{1}^{2} g_{2}^{4}-\frac{1677}{200} g_{1}^{4} g_{2}^{2}-\frac{3411}{1000} g_{1}^{6}-64 g_{3}^{2} y_{t}^{4}-\frac{16}{5} g_{1}^{2} y_{t}^{4}-\frac{9}{2} g_{2}^{4} y_{t}^{2} \\
& +10 \lambda\left(\frac{17}{20} g_{1}^{2}+\frac{9}{4} g_{2}^{2}+8 g_{3}^{2}\right) y_{t}^{2}-\frac{3}{5} g_{1}^{2}\left(\frac{57}{10} g_{1}^{2}-21 g_{2}^{2}\right) y_{t}^{2}-72 \lambda^{2} y_{t}^{2}+60 y_{t}^{6} .
\end{aligned}
$$


We calculate the Higgs boson pole mass $m_{H}$ from the running Higgs quartic coupling using the one-loop matching condition [13].

According to Eq. (2) there are additional contributions to the one and two loop beta function for $\lambda$ which are proportional to the $\kappa_{1}$ and $\kappa_{2}$ couplings. At one loop we have

$$
\delta \beta_{\lambda}^{(1)}=12\left(\kappa_{1}^{2}+\kappa_{2}^{2}\right) \lambda-12\left(\kappa_{1}^{4}+\kappa_{2}^{4}\right)
$$

and for two loop

$$
\begin{aligned}
\delta \beta_{\lambda}^{(2)}= & \left(\frac{8}{5} g_{1}^{2}-64 g_{3}^{2}\right)\left(\kappa_{1}^{4}+\kappa_{2}^{4}\right)-\frac{9}{2} g_{2}^{4}\left(\kappa_{1}^{2}+\kappa_{2}^{2}\right)+10 \lambda\left(\frac{1}{4} g_{1}^{2}+\frac{9}{4} g_{2}^{2}+8 g_{3}^{2}\right)\left(\kappa_{1}^{2}+\kappa_{2}^{2}\right) \\
& +\frac{3}{5} g_{1}^{2}\left(\frac{3}{2} g_{1}^{2}+9 g_{2}^{2}\right)\left(\kappa_{1}^{2}+\kappa_{2}^{2}\right)-72 \lambda^{2}\left(\kappa_{1}^{2}+\kappa_{2}^{2}\right)-3 \lambda\left(\kappa_{1}^{4}+\kappa_{2}^{4}\right)+60\left(\kappa_{1}^{6}+\kappa_{2}^{6}\right) .
\end{aligned}
$$

We next analyze the two loop RGEs numerically and show how the vacuum stability and perturbativity bounds on the SM Higgs boson mass are altered in the presence of the new $\mathrm{TeV}$ scale vectorlike particles.

We chose the cutoff scale to be $M_{\mathrm{GUT}}$, the scale at which the SM gauge couplings are all equal. This choice is motivated by the following argument. Namely, we want to have as much as possible model independent analysis and in the realistic GUT's we can have very different representation for fields. For instance there are many choice of fileds to break GUT symmetry [14], or if one address the question of flavor structure of fermions in the framework of GUT, or origin of neutrino mass and etc. Also it is well known that in many GUT the cutoff scale has to be very close to the $M_{\mathrm{GUT}}$ scale doe to existence of big representation under the GUT gauge symmetry, for instance in $\mathrm{SO}(10), \mathrm{E}(6)$ etc.

We define the vacuum stability bound as the lowest Higgs boson mass obtained from the running of the Higgs quartic coupling which satisfies the condition $\lambda(\mu) \geq 0$, for any scale between $M_{Z} \leq \mu \leq M_{\mathrm{GUT}}$. On the other hand, the perturbativity bound is defined as the highest Higgs boson mass obtained from the running of the Higgs quartic coupling with the condition $\lambda(\mu) \leq 4 \pi$ for any scale between $M_{Z} \leq \mu \leq M_{\mathrm{GUT}}$.

In Figure 1, we present the evolution of the gauge couplings for the SM (left panel) and for the extended SM (ESM) containing the vectorlike fermions $Q+\bar{Q}+D+\bar{D}$ (right panel). As noted in [5], in ESM model with new vectorlike fermions weighing a $100 \mathrm{GeV}$ or so, one can realize essentially perfect gauge coupling unification at some scale $M_{\mathrm{GUT}}$. Furthermore, if we require gauge coupling unification at a level of around $1 \%$ or so, then the new vectorlike fermion mass should weigh less than a TeV. For definiteness, we set $M_{F}=500 \mathrm{GeV}$ in our calculation. In this case the SM gauge couplings are unified at $M_{\mathrm{GUT}} \simeq 3 \times 10^{16} \mathrm{GeV}$. As seen in Figure 1, the new vectorlike particles help achieve unification by altering the slopes of the 

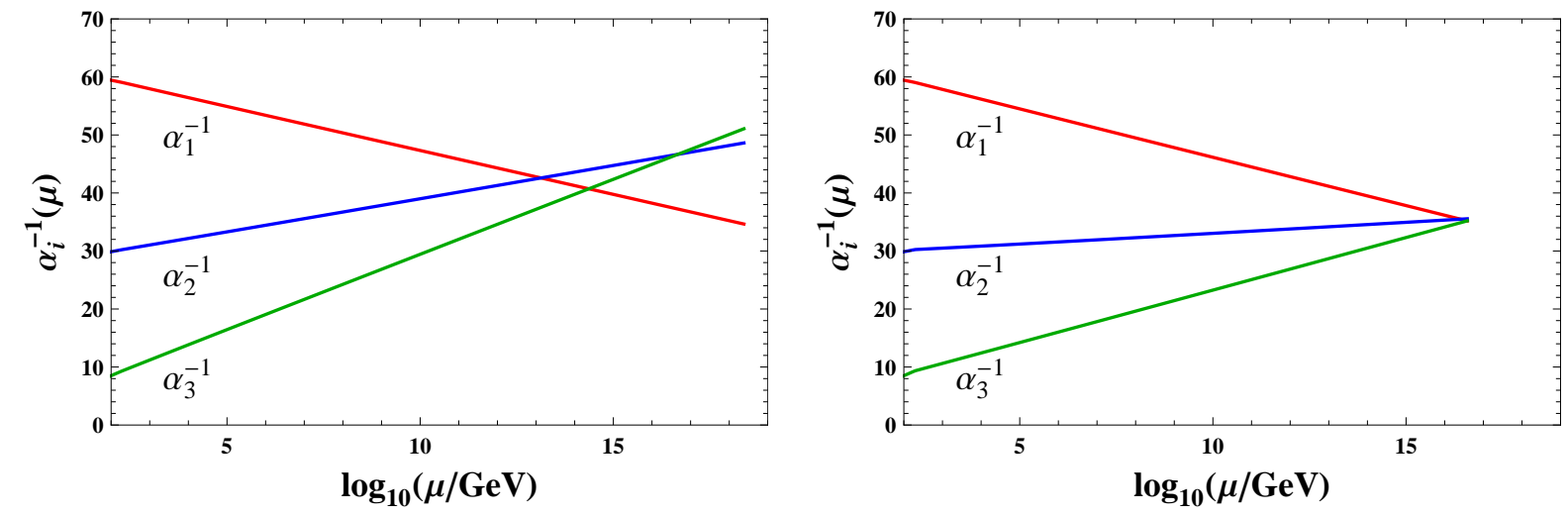

Figure 1: Gauge coupling evolution in the SM (left panel) and in the extended SM (right panel). The vectorlike mass is set equal to $500 \mathrm{GeV}$ and the gauge coupling unification scale is $M_{G U T} \simeq 3 \times 10^{16}$ $\mathrm{GeV}$.

three gauge couplings. In particular, the slope of $\alpha_{3}$ is changed and it becomes larger at $M_{\mathrm{GUT}}$ in comparison to the SM case. The evolution of the top Yukawa coupling is also affected and its value is somewhat smaller at $M_{\mathrm{GUT}}$.

In Figure 2 we show how the evolution of the two-loop top Yukawa coupling in ESM with $M_{F}=500 \mathrm{GeV}$. The red dashed line stands for the SM case, and the blue solid line corresponds to the ESM with $\kappa_{i}=0$. We also present in Figure 2 the evolution of the Higgs quartic coupling. The red dashed line corresponds to the vacuum stability bound for Higgs quartic coupling in the SM, and the blue solid line corresponds to the quartic couplings in the ESM. We see that at $M_{\mathrm{GUT}}$, the top Yukawa coupling in the ESM is smaller in comparison to the SM case. On the other hand, it is well known that in the determination of the SM Higgs boson mass vacuum stability bound [1], a crucial role is played by the interplay between the top Yukawa coupling and Higgs quartic coupling, which have comparable and dominant contributions in the RGE for Higgs quartic coupling (See Eq. (17)). The negative sign contribution from the top Yukawa coupling makes the Higgs quartic coupling smaller during the evolution. This is how the lower bound for Higgs boson mass is obtained in the SM. So, having in the model a smaller value at $M_{\mathrm{GUT}}$ for the top Yukawa coupling means having a milder contribution in the RGE for the Higgs quartic coupling, and this explains why in ESM, somewhat smaller values for the Higgs quartic coupling 3 can satisfy the vacuum stability bound, compared to the SM. In ESM, the lower bound for the SM Higgs boson mass using the one-loop matching condition [13] is found to be $m_{H}=117 \mathrm{GeV}$, close to the LEP bound of $114.4 \mathrm{GeV}$ [16]. We estimate a theoretical

\footnotetext{
${ }^{3}$ A similar observation was made in ref. 15] when considering the type III seesaw mechanism for neutrinos.
} 


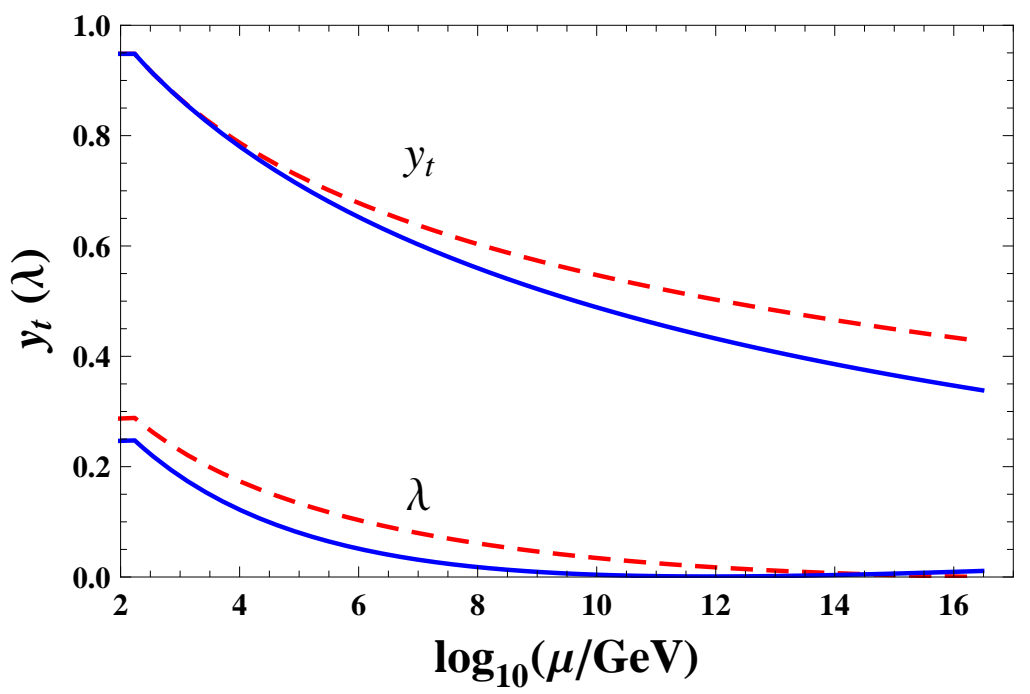

Figure 2: Evolution of the top Yukawa coupling in the SM (red dashed line) and in the extended SM (blue solid line). The evolution of the SM Higgs quartic coupling in the two cases are also displayed. We have set $M_{F}=500 \mathrm{GeV}$ and $\kappa_{i}=0$.

error in this prediction of about $2 \mathrm{GeV}$, which is in addition to the errors arising from the experimental uncertainties in the determination of the top quark mass and $\alpha_{3}$ [17].

As mentioned earlier, the $\kappa_{i}$ coupling in Eq. (2) can be $O(1)$ if $M_{F}>500 \mathrm{GeV}$. In Figure 3 we present the Higgs boson mass versus $\kappa_{i}$ for varying $M_{F}$ scales. For simplicity, we assume that $\kappa \equiv \kappa_{1}=\kappa_{2}$. The upper solid blue and red curves correspond to the Higgs perturbativity bound, and the lower dashed curves correspond to the vacuum stability bound when the vectorlike particle mass is taken to be $500 \mathrm{GeV}$ (dashed red) and $1 \mathrm{TeV}$ (dashed blue). It is interesting to observe that the perturbativity bound decreases as $\kappa$ increases from zero to $\kappa \approx 0.6$, and then increases as the value of $\kappa$ is increased further. We can easily understand this behavior at one loop level. It arises from the interplay between the terms $12 \lambda\left(\kappa_{1}^{2}+\kappa_{2}^{2}\right)$ and $-12\left(\kappa_{1}^{4}+\kappa_{2}^{4}\right)$ in Eq. (19). Up to $\kappa \approx 0.6$, the term proportional to $\kappa^{2} \lambda$ dominates over the $\sim \kappa^{4}$ contribution. So, for $\kappa \lesssim 0.6$, in the RGE in Eq. (16), we have an effective additional contribution with the same sign as the $\lambda$ coupling, which leads to the decrease of the perturbativity bound. For $\kappa \gtrsim 0.6$ the $\sim \kappa^{4}$ contribution dominates compared to the term $\kappa^{2} \lambda$, and we have an effective additional contribution which has the same sign contribution as the top quark in Eq. (16). This leads to an increasing perturbativity bound as the $\kappa$ coupling increases. Note that we have an upper bound $\kappa=0.86$ for $M_{F}=500 \mathrm{GeV}$, and $\kappa=0.84$ for $M_{F}=1 \mathrm{TeV}$. This happens because either the top Yukawa or $\kappa$ coupling becomes nonperturbative before the GUT scale. Corresponding to the upper bound for $\kappa$ couplings, we have an upper bound on the Higgs mass: $m_{H}=191$ $\mathrm{GeV}$ if $M_{F}=500 \mathrm{GeV}$, and $m_{H}=189 \mathrm{GeV}$ if $M_{F}=1 \mathrm{TeV}$. 


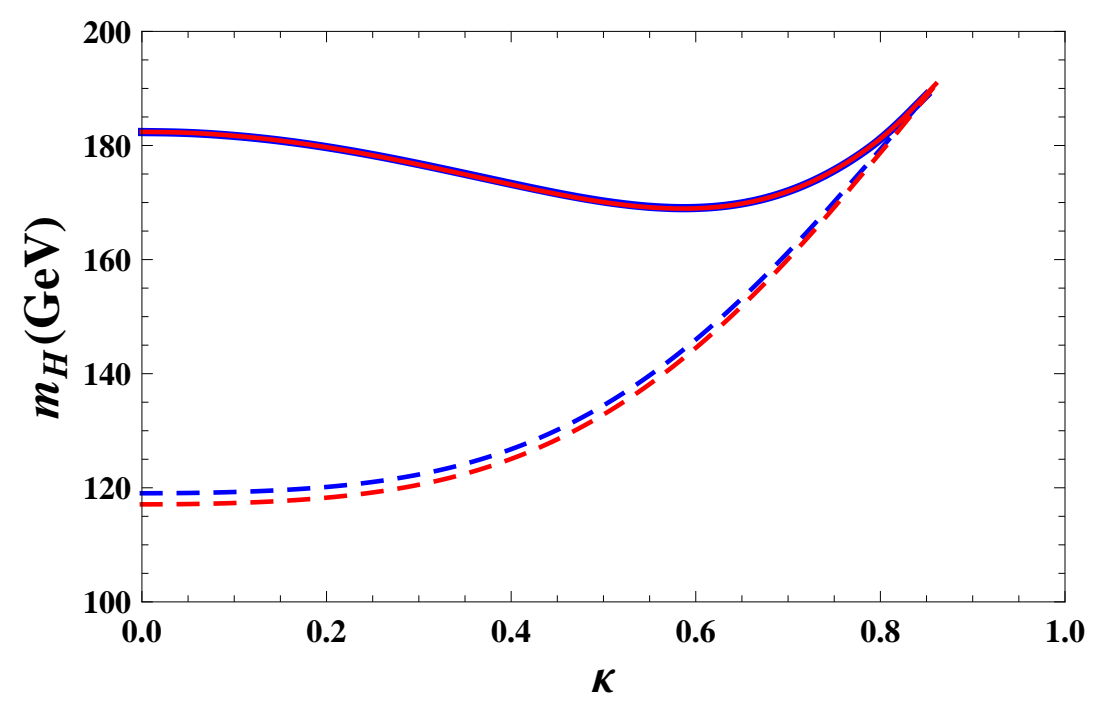

Figure 3: Perturbativity (solid) and vacuum stability (dashed) bounds on the Higgs boson pole mass $\left(m_{H}\right)$ versus $\kappa\left(\equiv \kappa_{1}=\kappa_{2}\right)$, with vectorlike particle mass $M_{F}=500 \mathrm{GeV}$ (red lines) and $M_{F}=1 \mathrm{TeV}$ (blue lines). The maximum value for the perturbativity bound is $m_{H} \simeq 191 \mathrm{GeV}$ when $\kappa=0.86$. The lower bound for the Higgs mass is $m_{H} \simeq 117 \mathrm{GeV}$, with $\kappa=0$ and $M_{F}=500 \mathrm{GeV}$.

We see in Figure 3 that the vacuum stability bound gradually increases as the $\kappa$ coupling increases. This happens because in the evolution of the Higgs quartic coupling, corresponding to the vacuum stability bound, the contribution proportional to the term $-\kappa^{4}$ dominates over the $\kappa^{2} \lambda$ contribution for lower values of $\kappa$. So in the RGE for the Higgs quartic coupling (see Eq. (16)) we have an additional contribution with the same sign as the top quark. This leads to the explanation why the vacuum stability bound increases when value of $\kappa$ increases at low scale, and they eventually merge with the vacuum stability bound. We obtain the following results for the Higgs mass corresponding to the vacuum stability bound: $m_{H}=117 \mathrm{GeV}$ when $M_{F}=500 \mathrm{GeV}$, and $m_{H}=119 \mathrm{GeV}$ when $M_{F}=1 \mathrm{TeV}$, with $\kappa=0$.

\section{Type I Seesaw and the Higgs Boson Mass}

We next consider the impact of type I seesaw physics [6] on the Higgs mass bounds found in the previous section. The terms relevant for neutrino oscillations through type I seesaw are given by

$$
\mathcal{L}_{\nu}=-y_{D}^{i j} l_{i} \nu_{j}^{c} \Phi^{c}-\frac{1}{2} M_{R}^{i j}\left(\nu^{c}\right)_{i}^{T} \nu_{j}+\text { h.c. }, \quad i, j=1,2,3 .
$$

Here $l_{i}$ is the lepton doublet, $\nu_{i}^{c}$ the right handed neutrino, $y_{D}^{i j}$ is neutrino Yukawa coupling and $M_{R}^{i j}$ denotes the right handed neutrino mass matrix. 


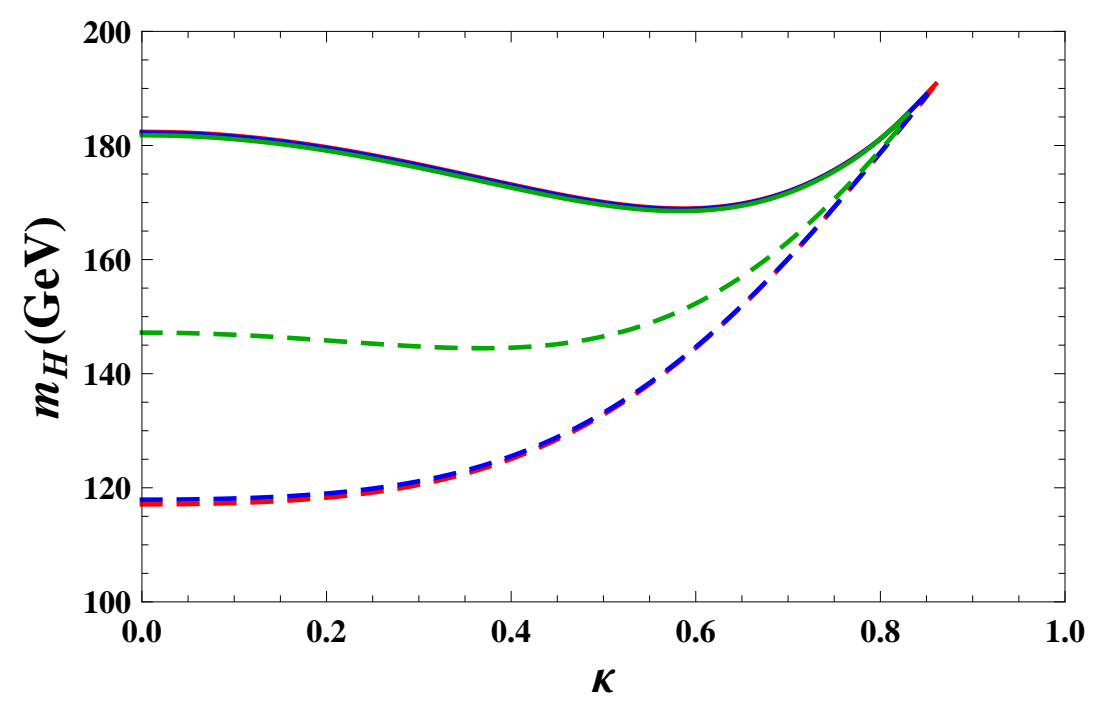

Figure 4: Perturbativity (solid) and vacuum stability (dashed) bounds on the Higgs boson pole mass $\left(m_{H}\right)$ versus $\kappa\left(\equiv \kappa_{1}=\kappa_{2}\right)$ in the extended SM, including type I seesaw physics. We consider three different type I seesaw scales $M_{R}=10^{13} \mathrm{GeV}$ (red), $10^{14} \mathrm{GeV}$ (blue) and $10^{15} \mathrm{GeV}$ (green). For our calculation we consider a hierarchical neutrino mass spectrum, and we set $M_{F}=500 \mathrm{GeV}$. The maximum and minimal values for the Higgs mass corresponding to the perturbativity and vacuum stability bounds are the same as in Figure 3.

Above the scale $M_{R}$ we have the following one loop RGE for $Y_{\nu} \equiv y_{D}^{i j}$,

$$
\frac{d \mathbf{Y}_{\nu}}{d \ln \mu}=\frac{1}{16 \pi^{2}} \mathbf{Y}_{\nu}\left(3 y_{t}^{2}+\operatorname{tr}\left[Y_{\nu}^{\dagger} Y_{\nu}\right]+\frac{3}{2} Y_{\nu}^{\dagger} Y_{\nu}-\left(\frac{9}{20} g_{1}^{2}+\frac{9}{4} g_{2}^{2}\right)\right)
$$

The various beta functions are modified as follows:

$$
\begin{aligned}
& \beta_{t}^{(1)} \rightarrow \beta_{t}^{(1)}+\operatorname{tr}\left[Y_{\nu}^{\dagger} Y_{\nu}\right] \\
& \beta_{\kappa_{1}}^{(1)} \rightarrow \beta_{\kappa_{1}}^{(1)}+\operatorname{tr}\left[Y_{\nu}^{\dagger} Y_{\nu}\right] \\
& \beta_{\kappa_{2}}^{(1)} \rightarrow \beta_{\kappa_{2}}^{(1)}+\operatorname{tr}\left[Y_{\nu}^{\dagger} Y_{\nu}\right] \\
& \beta_{\lambda}^{(1)} \rightarrow \beta_{\lambda}^{(1)}+4 \operatorname{tr}\left[Y_{\nu}^{\dagger} Y_{\nu}\right] \lambda-4 \operatorname{tr}\left[\left(Y_{\nu}^{\dagger} Y_{\nu}\right)^{2}\right] .
\end{aligned}
$$

It is certainly interesting to consider realistic cases of the neutrino mass matrix and mixing which reproduce the current neutrino oscillation data. We will consider a scenario in which the light neutrinos form a hierarchical mass spectrum. It was shown in Ref. [15] that the impact on the SM Higgs boson mass from an inverted-hierarchial neutrino mass spectrum is not significantly different from the hierarchial case.

The light neutrino mass matrix is diagonalized by a mixing matrix $U_{M N S}$ such that

$$
\mathbf{M}_{\nu}=\frac{v^{2}}{2 M} Y^{T} Y=U_{M N S} D_{\nu} U_{M N S}^{T},
$$


with $D_{\nu}=\operatorname{diag}\left(m_{1}, m_{2}, m_{3}\right)$, where we have assumed, for simplicity, that the Yukawa matrix $\mathbf{Y}_{\nu}$ is real. We further assume that the mixing matrix has the so-called tri-bimaximal form [18]

$$
U_{M N S}=\left(\begin{array}{ccc}
\sqrt{\frac{2}{3}} & \sqrt{\frac{1}{3}} & 0 \\
-\sqrt{\frac{1}{6}} & \sqrt{\frac{1}{3}} & \sqrt{\frac{1}{2}} \\
-\sqrt{\frac{1}{6}} & \sqrt{\frac{1}{3}} & -\sqrt{\frac{1}{2}}
\end{array}\right),
$$

which is in very good agreement with the current best fit values of the neutrino oscillation data [19].

For the hierarchical case the diagonal neutrino mass matrix is given by

$$
D_{\nu} \simeq \operatorname{diag}\left(0, \sqrt{\Delta m_{12}^{2}}, \sqrt{\Delta m_{23}^{2}}\right)
$$

We fix the input values for the solar and atmospheric neutrino oscillation data as [19]

$$
\begin{aligned}
& \Delta m_{12}^{2}=8.2 \times 10^{-5} \mathrm{eV}^{2} \\
& \Delta m_{23}^{2}=2.4 \times 10^{-3} \mathrm{eV}^{2}
\end{aligned}
$$

Our finding are presented in Figure 4 where we plot the vacuum stability (dashed) and perturbativity (solid) bound versus $\kappa\left(\equiv \kappa_{1}=\kappa_{2}\right)$, with $M_{F}$ set equal to $500 \mathrm{GeV}$. We consider three distinct mass scales for the heavy right handed neutrinos, namely, $M_{R}=10^{13} \mathrm{GeV}$ (red), $10^{14} \mathrm{GeV}$ (blue) and $10^{15} \mathrm{GeV}$ (green). The general picture of the Higgs mass versus $\kappa$ coupling is qualitatively the same as in Figure 3. Only the initial values for the Higgs mass when $\kappa=0$ is taken are changed depending on the type I seesaw scale. According to Eq. (23) the Dirac neutrino Yukawa coupling $Y_{\nu}$ gives an additional contribution to the Higgs quartic coupling RGE with the same sign as the top quark contribution. It is natural to expect that the vacuum stability bound will increase if the $Y_{\nu}$ coupling is increased. For $M_{R}=10^{13} \mathrm{GeV}$, the vacuum stability bound essentially coincides with the corresponding bounds in Figure 3. With $M_{R}=10^{14} \mathrm{GeV}$ the vacuum stability bound is only slightly altered since $Y_{\nu}$ is still not large at that scale in comparison to the top Yukawa coupling. For $M_{R}=10^{15} \mathrm{GeV}$ we see a significant change in the vacuum stability bound since now the coupling $Y_{\nu}$ is larger than the top Yukawa coupling, and the two of them together force the Higgs quartic coupling at low scale to be larger in order to satisfy the vacuum stability bound. Note that there is hardly any impact of type I seesaw on the perturbativity bound. This is due to the fact that above the seesaw scale the Higgs quartic coupling is already larger than $Y_{\nu}$. 


\section{Conclusion}

Following ref. [5], we have considered a plausible extension of the SM in which new vectorlike fermions carrying SM quantum numbers and with masses of order $300 \mathrm{GeV}-1 \mathrm{TeV}$ are introduced. This relatively modest extension of the SM, denoted by ESM in the text, leads to a rather precise unification of the SM gauge couplings at $M_{\mathrm{GUT}} \sim 3 \times 10^{16} \mathrm{GeV}$, and it also gives rise to a vacuum stability bound on the SM Higgs mass of $117 \mathrm{GeV}$. The perturbativity bound on the Higgs mass is estimated to lie close to $190 \mathrm{GeV}$. The new vectorlike fermions should be accessible at the LHC.

\section{Acknowledgments}

We thank Yukihiro Mimura, Nobuchika Okada, Mansoor Ur Rehman and Joshua R. Wickman for useful comments and discussion. This work is supported in part by the DOE Grant \#DEFG02-91ER40626 (I.G. H.B. and Q.S.), GNSF grant 07_462_4-270 (I.G.) and by Bartol Research Institute (B.H.).

\section{References}

[1] N. Cabibbo, L. Maiani, G. Parisi and R. Petronzio, Nucl. Phys. B 158, 295 (1979); P. Q. Hung, Phys. Rev. Lett. 42, 873 (1979); M.A.B. Beg, C. Panagiotakopoulos and A. Sirlin, Phys. Rev. Lett.52 (1984) 883; K. S. Babu and E. Ma, Phys. Rev. Lett. 55, 3005 (1985); M. Lindner, Z. Phys. C 31, 295 (1986); M. Sher, Phys. Rept. 179, 273 (1989); G. Altarelli and G. Isidori, Phys. Lett. B 337, 141 (1994); J. A. Casas, J. R. Espinosa and M. Quiros, Phys. Lett. B 342, 171 (1995); Phys. Lett. B 382, 374 (1996); J. R. Espinosa and M. Quiros, Phys. Lett. B 353, 257 (1995); P. Q. Hung and G. Isidori, Phys. Lett. B 402, 122 (1997); J. Ellis, J. R. Espinosa, G. F. Giudice, A. Hoecker and A. Riotto, Phys. Lett. B 679, 369 (2009).

[2] I. Gogoladze, N. Okada and Q. Shafi, Phys. Rev. D 78, 085005 (2008).

[3] G. Lazarides, Q. Shafi and C. Wetterich, Nucl. Phys. B181, 287 (1981); R. N. Mohapatra and G. Senjanović, Phys. Rev. D 23, 165 (1981); M. Magg and C. Wetterich, Phys. Lett. B 94, 61 (1980); J. Schechter and J. W. F. Valle, Phys. Rev. D 22, 2227 (1980).

[4] N. Haba, S. Matsumoto, N. Okada and T. Yamashita, JHEP 0602, 073 (2006); I. Gogoladze, N. Okada and Q. Shafi, Phys. Lett. B 655, 257 (2007); I. Gogoladze, N. Okada 
and Q. Shafi, Phys. Lett. B 659, 316 (2008); Y. Hosotani, N. Maru, K. Takenaga and T. Yamashita, Prog. Theor. Phys. 118, 1053 (2007).

[5] U. Amaldi, W. de Boer, P. H. Frampton, H. Furstenau and J. T. Liu, Phys. Lett. B 281, 374 (1992); J. L. Chkareuli, I. G. Gogoladze and A. B. Kobakhidze, Phys. Lett. B 340 (1994) 63; J. L. Chkareuli, I. G. Gogoladze and A. B. Kobakhidze, Phys. Lett. B 376, 111 (1996); D. Choudhury, T. M. P. Tait and C. E. M. Wagner, Phys. Rev. D 65, 053002 (2002); D. E. Morrissey and C. E. M. Wagner, Phys. Rev. D 69, 053001 (2004).

[6] P. Minkowski, Phys. Lett. B 67, 421 (1977); T. Yanagida, in Proceedings of the Workshop on the Unified Theory and the Baryon Number in the Universe (O. Sawada and A. Sugamoto, eds.), KEK, Tsukuba, Japan, 1979, p. 95; M. Gell-Mann, P. Ramond, and R. Slansky, Supergravity (P. van Nieuwenhuizen et al. eds.), North Holland, Amsterdam, 1979, p. 315; S. L. Glashow, The future of elementary particle physics, in Proceedings of the 1979 Cargèse Summer Institute on Quarks and Leptons (M. Lévy et al. eds.), Plenum Press, New York, 1980, p. 687; R. N. Mohapatra and G. Senjanović, Phys. Rev. Lett. 44, 912 (1980).

[7] G. Barenboim, F. J. Botella and O. Vives, Nucl. Phys. B 613, 285 (2001); D. Choudhury et al. in ref [5].

[8] L. Lavoura and J.P. Silva, Phys. Rev. D 47, 2046 (1993); N. Maekawa, Phys. Rev. D 52, 1684 (1995).

[9] C. Amsler et al. [Particle Data Group], Phys. Lett. B 667, 1 (2008).

[10] M. E. Machacek and M. T. Vaughn, Nucl. Phys. B 222, 83 (1983); Nucl. Phys. B 236, 221 (1984); Nucl. Phys. B 249, 70 (1985); C. Ford, I. Jack and D. R. T. Jones, Nucl. Phys. B387 (1992) 373, [Erratum-ibid. B504 (1997) 551]; H. Arason, D. J. Castano, B. Keszthelyi, S. Mikaelian, E. J. Piard, P. Ramond and B. D. Wright, Phys. Rev. D 46, 3945 (1992); V. D. Barger, M. S. Berger and P. Ohmann, Phys. Rev. D 47, 1093 (1993); M. X. Luo and Y. Xiao, Phys. Rev. Lett. 90, 011601 (2003).

[11] See, for example, H. Arason, D. J. Castano, B. Keszthelyi, S. Mikaelian, E. J. Piard, P. Ramond and B. D. Wright, Phys. Rev. D 46, 3945 (1992); H. E. Haber, R. Hempfling and A. H. Hoang, Z. Phys. C 75, 539 (1997).

[12] See, for example, F. Jegerlehner, M. Y. Kalmykov and O. Veretin, Nucl. Phys. B 641, 285 (2002); Nucl. Phys. B 658, 49 (2003); F. Jegerlehner and M. Y. Kalmykov, Nucl. Phys. B 676, 365 (2004). 
[13] A. Sirlin and R. Zucchini, Nucl. Phys. B 266 (1986) 389.

[14] See for instance, P. Nath and P. Fileviez Perez, Phys. Rept. 441, 191 (2007).

[15] J. A. Casas, V. Di Clemente, A. Ibarra and M. Quiros, Phys. Rev. D 62, 053005 (2000); I. Gogoladze, N. Okada and Q. Shafi, Phys. Lett. B 668, 121 (2008).

[16] R. Barate et al. [LEP Working Group for Higgs boson searches], Phys. Lett. B 565, 61 (2003).

[17] See for instance, J. R. Espinosa, G. F. Giudice and A. Riotto, JCAP 0805, 002 (2008).

[18] P. F. Harrison, D. H. Perkins, W. G. Scott, Phys. Lett. B530 167 (2002).

[19] B. T. Cleveland et.al, Astrophys.J. 496505 (1998); Super-Kamiokande Collaboration, Phys. Lett. B539 179 (2002); Super-Kamiokande Collaboration, Phys. Rev. D71 112005 (2005); M. Maltoni, T. Schwetz, M.A. Tortola, J.W.F. Valle New J.Phys. 6122 (2004); A. Bandyopadhyay et al, Phys. Lett. B608 115 (2005); G. L. Fogli et al, Prog. Part. Nucl. Phys. 57742 (2006); For a recent review, see, for example, H. Nunokawa, S. J. Parke and J. W. F. Valle, Prog. Part. Nucl. Phys. 60, 338 (2008). 